DOI: $10.14746 /$ por.2019.1.11

\title{
BUDDENBROOKS AM SCHWARZEN MEER. VLADIMIR JABOTINSKYS ODESSA-ROMAN DIE FÜNF
}

\author{
IRMELA VON DER LÜHE \\ (Freie Universität Berlin)
}

\begin{abstract}
Schlüsselwörter: Jüdischer Familienroman, Odessa als umstrittener Erinnerungsort, Symbolismus, Impressionismus, Straßen, Plätze und Orte als Räume der Erinnerung

Keywords: Jewish familiy novel, Odessa as a controversial space of memory, symbolism, impressionism, streets, places and rooms of memory
\end{abstract}

\begin{abstract}
Irmela von der Lühe, BUDDENBROOKS AM SCHWARZEN MEER. VLADIMIR JABOTINSKYS ODESSA-ROMAN DIE FÜNF. „PORÓWNANIA” 1 (24), 2019. T. XXIV, S. 123-133. ISSN 1733-165X. Vladimir Jabotinskys Roman Die Fünf ist bei seinem Erscheinen in Deutschland (2012) als eine literarische Entdeckung gefeiert worden. In der Familiengeschichte der Milgroms aus dem Odessa der Jahre vor der Revolution von 1905 spiegeln sich Hoffnungen und Enttäuschungen der Revolution und der jüdischen Assimiliation, des Aufbruchs in eine neue Welt der Moderne und des Absturzes in eine Welt der brutalen Antimoderne. Der Beitrag untersucht die besonderen literarischen Strategien einer Arbeit an der Erinnerung: des Erzählens von Straßen, Plätzen und Räumen, der Analogisierung des politischen Geschehens mit einem Bühnengeschehen, der symbolistischen Überhöhung der Realität durch Rückgriff auf literarische Texte. Nicht nur aus politischen, auch aus erzählerischen Gründen wird Odessa in Jabotinkskys Roman also zu einem umstrittenen Erinnerungsort.
\end{abstract}

Abstract: Irmela von der Lühe, BUDDENBROOKS AT THE BLACK SEA. VLADIMIR JABOTIN-
SKYS ODESSA-NOVEL THE FIVE. "PORÓWNANIA" 1 (24), 2019. Vol. XXIV, P. 123-133. ISSN
1733-165X. When Jabotinksys novel first appeared in Germany (2012) it was celebrated as a spec-
tacular literary event. Jobtinsky's Jewish family novel is situated in the decades before the rev-
olution of 1905; it deals with revolutionary hope and disappointment, with Jewish assimiliation
and the idea of a new liberal and tolerant world. It ends with a complete decline of these visions
into brutal antimodernism. This article describes special narrative strategies in producing spaces

1 E-mail:vdluehe@zedat.fu-berlin.de 
of memory as streets, places and special public locations. Thereby political events appear as analogies to theater plays and also in a symbolistic framework. By this Jabotinskys novel has become a controversial space of memory not only for political but also literary reasons.

In welchem Maße die 1794 auf Anweisung Katharinas der Großen gegründete Hafenstadt Odessa ein „umstrittener Erinnerungsort" ist, bedarf kaum einer Erläuterung (Raabe 2009). So sehr man sich im Anschluss an Pierre Noras große Arbeit über Erinnerungsorte an einen metaphorischen Gebrauch dieses Terminus gewöhnt hat, so dass Personen und Familien, Mythen, Kunstwerke und politische Ereignisse zu Gedächtnisorten, zu Fixpunkten des kollektiven und kommunikativen Gedächtnisses werden, so eindeutig ist doch die topographische und historisch-politische Bedeutung des Begriffs im Falle von Odessa.

Die russische Annexion der Krim im Jahre 2014 hat diese Eindeutigkeit schmerzhaft zur Gegenwart werden lassen.

Und doch scheint die große, einst multiethnische und multikulturelle Hafenstadt am Schwarzen Meer in der Kultur-und Literaturgeschichte und damit in einem kulturellen Gedächtnis, das sich insbesondere den Verbrechen und politischen Verwerfungen des 20. Jahrhunderts verpflichtet sieht, von ihrer Faszination nichts eingebüßt zu haben; im Gegenteil, es entsteht der Eindruck, dass literarische Texte, die wie im vorliegenden Falle im Odessa der Revolution von 1905 situiert sind, eine Form der Erinnerung ermöglichen, die zwischen Nostalgie und Sentimentalität, Wehmut und Kitsch, aber auch zwischen kritischer Reflexion und analytischer Präzision eine ganz eigene Balance halten. Für den hier interessierenden Text und seinen Autor gilt indes noch mehr. Er löste in Feuilleton und Öffentlichkeit eine nachgerade enthusiastische Reaktion aus, was wohl auch dem Umstand geschuldet ist, dass beide (Text und Autor) bis 2012 in Deutschland entweder vollständig unbekannt oder vergessen waren.

Dabei erwies es sich offenbar als höchst wirksam, für einen Roman, der seit 1933 zunächst in Fortsetzungen in der Pariser russisch-jüdischen Zeitschrift „Rasswyet" („Morgenröte“) und 1936 als Buch unter dem Titel „Die Fünf“ erschienen war, mit der Behauptung zu werben, es handele sich um die Buddenbrooks vom Schwarzen Meer (Jabotinsky 2017).

Zwar entwirft Jabotinsky in der Tat ein Gesellschaftspanorama, und zwar im Muster eines jüdischen Familienromans, und es geht auch um eine Verlust-und Untergangsgeschichte. Damit sind die Analogien zu Thomas Manns Roman über den „Verfall einer Familie“ aber auch schon erschöpft. Denn Zerstörung und Untergang verdanken sich bei Jabotinsky gerade nicht einer epochentypischen Verschränkung von kaufmännischen Fehlentscheidungen, überzogenem Pflichtethos und todessüchtigem Kult von Schönheit und Kreativität, für die wiederum die Musik Richard Wagners und die Philosophie Arthur Schopenhauers Pate standen. Das Ende der in Odessa ansässigen assimilierten jüdischen Familie Milgrom und ihrer fünf Kin- 
der ist keineswegs Ausdruck eines inneren Auflösungsprozesses aufgrund schwindender Kräfte, verweigerten Leistungsdenkens und selbstvergessener Verwöhntheit und Weltferne. Es steht in direktem Zusammenhang mit Vorgeschichte und Verlauf der Revolutionsereignisse des Jahres 1905, mit mentalen und diskurspolitischen Entwicklungen im Russland seit Ende des 19. Jahrhunderts, mit Debatten um Kommunismus und Zionismus, Revolution und Assimilation. Der Vergleich mit Thomas Manns dekadenzkritischem Familienroman führt also nicht sehr weit.

Ich frage daher im Folgenden nach erzählerischen Besonderheiten und narrativen Strategien, die Jabotinskys Odessa-Roman zum Erinnerungsort, womöglich zu einem "umstrittenen“, vor allem aber zu einem Ort der Erinnerung an modernetypische Konfliktkonstellationen um jüdische Identität und Selbstwahrnehmung machen.

Ich schicke einige Hinweise zum Autor voraus, dessen Wiederentdeckung im deutschen Sprachraum auch deswegen so spektakulär verlief, weil er im Bereich der jüdischen Geschichte, insbesondere der Forschung zur Geschichte des Zionismus zwar als Journalist und Vertreter radikal zionistischer Bestrebungen, nicht aber als Romanautor beachtet worden war. Der begnadete politischer Redner, der vermutlich nicht nur für Arthur Koestler "der erste politische Schamane meines Lebens" (Buckard 75) wurde, hatte als Gründer der "jüdischen Legion“ im Ersten Weltkrieg und seit 1937 als Oberkommandierender des Irgun, einer Terrororganisation in Palästina, die Attentate auf Araber und Briten verübte, von sich reden gemacht. Als zionistischer Revisionist war er bereits 1923 mit seinem Essay „The Iron Wall“ für eine offensive jüdische Besiedelung Palästinas und den rücksichtslosen Kampf gegen die dort lebenden Araber eingetreten (Awerbuch 137+261 f.).

Nicht ohne Grund wird in der auf Jabotinsky zurück gehenden Metapher von der „Eisernen Mauer aus jüdischen Bajonetten“ noch immer die Grundlegung der heutigen israelischen Politik gegenüber den Palästinensern gesehen.

1880 in Odessa geboren und 1940 in New York gestorben, hatte Jabotinsky in Rom Rechtswissenschaften studiert, anschließend unter dem Pseudonym "Altalena" (Kaplan 2011) in Odessa für verschiedene Zeitungen geschrieben, 1911 in Odessa einen Verlag für hebräische Literatur gegründet. Er hat Dantes Inferno ins Hebräische und Chaim Nachman Bialiks Gedicht In der Stadt des Schlachtens aus dem Hebräischen ins Russische übersetzt. Der aus einer bürgerlichen Familie stammende und orthodox erzogene Jabotinsky entfernte sich zwar mehr und mehr vom orthodox-religiösen Judentum, wurde offenbar durch das Pogrom von Kischinew (Hohmann, Mümken 2015), dem Bialik mit dem erwähnten Gedicht ein großes lyrisches Denkmal setzte, zum radikalen Zionisten. Noch im Jahr dieses Schreckensgeschehens, 1903, nahm Jabotinsky am 6. Zionistenkongress in Basel teil, wurde mit Theodor Herzl bekannt und zu einem der wichtigsten und wirkungsvollsten Sprecher der russischen Juden. Freilich erschien ihm die Politik der WZO nicht konsequent und radikal genug, von zu großer Kompromissbereitschaft gegenüber der 
britischen Mandatsregierung geprägt. Seit Adolf Hitler am 30. Januar 1933 die politische Macht in Deutschland übertragen worden war, hat Jabotinsky immer wieder vor einer Katastrophe für das osteuropäische Judentum gewarnt; noch in den Monaten vor dem deutschen Überfall auf Polen hat er die Regierungen Polens, Rumäniens und Ungarns davon zu überzeugen versucht, eine Massenauswanderung von annähernd anderthalb Millionen europäischer Juden nach Palästina zu fördern; ein Vorschlag, der sowohl im polnischen als auch im amerikanischen Judentum auf entschiedene Ablehnung stieß. Als einen hellsichtigen Realisten und zugleich als einen „kosmopolitischen Ultra-Nationalisten“ hat man Jabotinsky daher aus guten Gründen bezeichnet (Stanislawski nach Brumlik 74).

Ich komme damit zu seinem Roman Die Fünf, dem man vorgeworfen hat, er sei „eher die Kopfgeburt eines Politikers als eine literarisch überzeugende Schöpfung" (Schmid 2013). Das freilich kann nur behaupten, wer die lakonisch-knappe, ironisch-anspielungsreiche, keineswegs dokumentarisch-didaktische Komposition dieses mal eben 250 Seiten umfassenden Textes ignoriert. Es dominiert ein Erzählen in Szenen, in kurzen Dialogen. Ihr Arrangeur ist ein sich erinnernder Erzähler, der als Journalist arbeitet und zum Personeninventar des Romans gehört. Als Freund der Familie Milgrom hatte er zu Beginn des 20. Jahrhunderts in ihrem Hause und mit ihren fünf Kindern freundschaftlich verkehrt. Aus beruflichen Gründen musste er Odessa immer mal wieder verlassen. Jahre später wird er zum Zeugen jenes Geschehens, das mit der atmosphärischen Vorgeschichte und dem brutalen Verlauf der Revolution von 1905, mit der Niederschlagung des Aufstands der Matrosen des Panzerkreuzers Potjomkin und Pogromen gegen die Juden Odessas verbunden ist.

Die ersten Sätze des Romans, aus dem Abstand von 30 Jahren aufgeschrieben, lauten:

Der Beginn dieser Erzählung aus dem Leben des vergangenen Odessa liegt ganz am Anfang unseres Jahrhunderts. Dessen erste Jahre heißen bei uns Frühling: ein gesellschaftliches und staatliches Erwachen, und für meine Generation fielen diese Jahre zusammen mit dem persönlichen Frühling der Jugend von Zwanzigjährigen (Jabotinsky 5).

„Dass auch der Frühling zur Massentragödie werden würde“ (Jabotinsky 63), nehmen Erzähler und Romanpersonal zwar wahr, aber „dass diese Tragödie von langer Dauer sein würde", das ahnte man nicht. Der Konnex zwischen Aufbruch und Tragödie, Frühling und Fieber wird zum Leitmotiv für eine Geschichte, die sich für den Erzähler retrospektiv als zugleich "fröhlich und bitter“ (39), "grauenhaft und erhaben" (38) darstellt.

Und doch wird sie nicht von den politischen Ereignissen her erzählt, sondern als Porträtgalerie der Milgrom-Familie und anderer Personen. Auch ist der Begriff Erzählen nur bedingt treffend, denn die meist kurzen 29 Kapitel sind häufig als Bühnenauftritte, als szenische Episoden mit satirischen oder auch romantisch-ly- 
rischen Einlagen, gelegentlich als Abfolge heiterer Anekdoten angelegt. Nicht zufällig beginnt das Geschehen denn auch im Theater Odessas: mit einer umjubelten Aufführung von Maurice Maeterlincks „Monna Vanna“ (1902); bekanntlich ein Programm-Stück des europäischen Symbolismus, dessen Heldin Giovanna im Konflikt zwischen zwei rivalisierenden Männern, in militärisch hoffnungsloser Lage einen Ausweg aus der ihr zugeschriebenen Rolle sucht und findet. Der Mann, dem sie aus politischen Gründen, der Rettung Pisas, zugeführt werden soll, entpuppt sich als ein Freund aus Kindertagen; nichts geschieht, aber der so gerettete Ehemann und Kommandant Pisas glaubt den Beteuerungen seiner Gattin nicht. Das Stück spielt zwar im 16. Jahrhundert, überhöht das militärisch-politische Geschehen aber psychologisch-symbolisch und arbeitet dabei mit einer gleich doppelten Referenz: auf Friedrich Hebbels „Judith“ und auf Leonardos berühmtem Bild "Mona Lisa“.

Ein solches an Situationen und nicht an Handlungen, an Gefühls- und nicht an Ereigniskonstellationen orientiertes Verfahren ist für Maeterlinck wie auch für den Symbolismus insgesamt charakteristisch; und charakteristisch ist nun vor allem, dass Jabotinsky es in eine Romanform bringt. Das Bauprinzip des Maeterlinck-Stückes, dessen Aufführung am Beginn des Romans ausführlich beschrieben und zum Ort der ersten Begegnung zwischen Frau Milgrom, ihrer ältesten Tochter, Marussja, und dem Erzähler wird, macht Jabotinksky also zum Konstruktionsprinzip seines Romans.

Freilich ist damit das durch „Monna Vanna“ gerettete Pisa des 16. Jahrhunderts keineswegs die historische Folie für das Odessa der Jahrhundertwende und des Revolutionsjahres 1905. Die Komposition des Romans weist zwar eine deutliche Nähe zu symbolistischen Verfahren auf und bezieht sich oft direkt auf dramatische und lyrische Texte aus der russischen und europäischen Literatur, daneben stehen aber filmische Prinzipien, wie sie durch Eisensteins Stummfilm über den Panzerkreuzer Potjomkin berühmt wurden. Es wäre zweifellos lohnend, dies im Einzelnen zu untersuchen. Für die Frage nach Strategien ästhetischer Erinnerungsarbeit ergibt sich fürs erste: Sie verdankt sich intertextuellen und intermedialen Anspielungen und Bezugnahmen, sie erfolgt nicht im Modus dokumentarisch-realistischen Erzählens, chronikalischer Aufzählung, geschichtsdidaktischer Anstrengung, sondern der assoziativen Verknüpfung von Alltagsereignissen und Zufallsbegebenheiten. Dabei entsteht kein auf Linearität und Geschlossenheit angelegtes Porträt einer Familie oder wohl gar ein Familienroman; es entsteht ein Mosaik von Odessa, das Gebäude und Milieus, Plätze und Straßenzüge, politische Geschehnisse und gesellschaftliche Skandale, vor allem aber die fünf Kinder der Familie Milgrom ineinander spiegelt.

Wie erwähnt beginnt alles im Stadttheater von Odessa. Dem Bericht über die Maeterlinck-Aufführung sind Beobachtungen im Zuschauerraum, schließlich ist ihm die Begegnung mit dem „rothaarige(n) Fräulein“ (9), mit Marussja, eingeschrieben; dabei kommt es noch gar nicht zu einem tatsächlichen Gespräch, sondern lediglich zum Protokoll von Gesprächsfetzen über das Stück und seine Heldin, die 
an das Ohr des Erzählers dringen. Das Bild von dieser ältesten Milgrom-Tochter entsteht also zunächst aus Fremdblicken und zitierten Äußerungen, darunter diejenige, die auch den Leser/die Leserin aufhorchen lässt. Denn Marussjas Kommentar zur Figur der Monna Vanna und der ihr abverlangten Rettungstat, die dann doch nicht erforderlich wurde und zu einer ganz anderen Krise führt, lautet: „Schrecklich! (...) ich anstelle von Monna Vanna hätte das niemals zugelassen. Was für ein Trottel!" (9).

Damit erntet Marussja natürlich große Bewunderung durch die umstehenden Studenten, von denen einer sich zu der Bemerkung hinreißen lässt:

„Sie sind wundervoll, Marussja, immer sagen Sie etwas, wofür ich Sie küssen möchte..." Die so Gelobte quittiert es gleichgültig-spöttisch, es gebe „auf der Deribassowka bald keinen einzigen Studenten mehr, der sich rühmen könnte, mich noch nie geküsst zu haben" (10).

Der schnoddrig-saloppe Ton ist charakteristisch für die älteste Milgrom-Tochter, die auch mit ihrer Mutter in entsprechender Diktion zu kommunizieren pflegt. Provokante sprachliche und habituelle Tabubrüche bestimmen den Tonfall fast aller Mitglieder der Familie; der dem Erzähler besonders nahestehende Bruder Serjosha artikuliert sich überdies besonders gern in versifizierter Form, und zwar in Gestalt gereimter Zweizeiler. Mit ihnen werden alle Familienmitglieder, aber auch jeder Besucher des gastfreundlichen Hauses Milgrom porträtiert. Der hoffnungsvolle Jung-Lyriker Serjosha ist noch Gymnasiast, raffinierter Schulschwänzer, handwerklich und intellektuell gleichermaßen begabt. Sein Elternhaus nennt er einen „Flohmarkt", den die Abendgäste seiner Schwester als „Passagiere“ frequentierten. Über seinen älteren Bruder Marko teilt er dem Erzähler mit, er sei „dieses Jahr Nietzscheaner" (16) und ergänzt die knappe Mitteilung durch den boshaften Zweizeiler: „Ideen nach Mode, doch zerrissene Kleider/Gelehrter Mann und dreifach Sitzenbleiber" (16). Über die andere, ebenfalls ältere Schwester Lika heißt es, sie habe „ihre Nägel bis auf den letzten abgekaut, und nun langweilt sie sich und ist wütend auf ganz Odessa“ (ebd.).

Der jüngste Bruder Torik schließlich sei „eine Stütze des Throns“, sein Urteil über alles „ist immer so korrekt, dass einem schon von weitem schlecht werden kann" (17).

Von den fünf Milgrom-Kindern wird dieser vernünftig korrekte Torik als einziger am Leben bleiben und zum Christentum konvertieren; ein Umstand, der die ohnehin schwer geschlagenen Eltern noch einmal hart trifft und den alten Vater zu einer ganz eigenen Hiob-Interpretation veranlassen wird.

Vom launig-frechen, lakonisch-knappen, anspielungsreich-präzisen Erzählstil des Romans, seines Erzählers und seiner Figuren war schon die Rede. Darüber hinaus basiert Jabotinskys literarische Erinnerungsarbeit auf topographischen, raumsemantischen Mitteln. Wie erwähnt lernen Erzähler und Leser wichtige Figuren des Romans, Mutter und Tochter Milgrom, als Zuschauer einer spektakulären Theater- 
aufführung kennen. Über Orte und Plätze, Straßen und Räume werden auch die weiteren 28 kurzen Erzählsequenzen inszeniert: Torik und Lika lernt man in und über ihre Privatzimmer kennen, ersteres ist voller Bücher („,die die verschiedenen Stadien seiner intellektuellen Entwicklung spiegelten") (44), dasjenige der asketisch-hasserfüllten jungen Kommunistin Lika ist von spartanischer Einfachheit, die einzige Zierde bildet ein riesiges Wandporträt von Ferdinand Lassalle. Der jährliche studentische Silvesterball findet im "Palast der Börse“ und dort in einem „Seitensaal“ statt, der den Namen "Totenkammer" trägt; dort hat nur ein "fortschrittlich gesinntes" Publikum Zugang, es dominiert der "Rausch der Worte und Ideen“. Armenier und Georgier, Polen, Zionisten und Bundisten treffen heftig aufeinander; meist endet die Debatte um Nationen und nationale Zugehörigkeit in albernen Trinksprüchen und proto-pathetischen Bekundungen, wie "wenn Sie schon eine Nation suchen, warum wollen Sie dann nicht Zionist werden“ (61).

Der Hausherr der Familie Milgrom hält sich meistens in seinem Kontor auf, möchte den Erzähler unbedingt für sein Geschäft gewinnen, weil der als Journalist keinen richtigen Beruf ausübe. Ignaz Albertowitsch stammt aus dem wolhynischen Ghetto und hat sich mit eiserner Disziplin als Getreidehändler nach oben gearbeitet. Den deutschen Klassikern gilt seine ganze Liebe. Mit Zitaten aus Werken Adalbert von Chamissos und Nikolaus Lenaus, vor allem aber Ludwig Börnes und anderer deutsch-jüdischer Autoren spickt er seine Reden und trägt auf diese Weise ,jenen Stempel, den wir mit dem lächerlichen Wort ,Intelligenzler' benennen“ (28).

Über Räume, aber eben auch über Autoren und Texte werden die Personen des Romans präsentiert, und natürlich dient dies alles der Illustration und Demontage zeit-und milieutypischer Attitüden und sozialcharakteristischer Verhaltensweisen. Dabei finden sich Schlussfolgerungen von bemerkenswerter Präzision, so etwa aus Anlass des Begriffs „Intelligenzler“: „ein echter Intelligenzler kann seelenruhig, ja gähnend offenbaren, dass er von Maupassant oder Hegel nicht die geringste Ahnung hat - es geht nicht um reale Eigenschaften, sondern um eine Art generelle Imprägnierung mit Kultur im Allgemeinen“ (28).

Man merkt an diesen Beispielen, die vielfach ergänzt werden könnten, dass der Roman einen geistreich-witzigen, sarkastisch-demontierenden Blick auf ein kulturelles, soziales, politisches und diskursives Gesamtgeschehen im Odessa vor und nach den Ereignissen des Jahres 1905 wirft. Alltägliches und Grundsätzliches, Banales und Systematisches steht ganz selbstverständlich und gleichrangig nebeneinander; von den persönlichen und von den politischen Katastrophen, vom tragischen Ungeschick, das zum Tode von Sohn Marko führt (er ertrinkt beim Versuch eine Frau vor dem Ertrinken zu retten) und von den absurd-hochstaplerischen Verhaltensweisen Serjoshas, der sich in eine ménage à trois begeben hatte und vom betrogenen Ehemann und Vater mit einer giftigen Säure verunstaltet wurde, schließlich von den schaurig-grotesken Umständen, durch die Marussja ums Leben kommt (sie verbrennt in der eigenen Küche): Von all diesen höchst ungewöhnlichen Todesum- 
ständen erfährt der Leser im gleichen Ton, wie von den blutigen Zusammenstößen im Odessa des Revolutionsjahres 1905, vom Aufeinandertreffen der jüdischen Volkswehr mit der städtischen Gendarmerie, vom Beschuss der Stadt durch die Matrosen des Panzerkreuzers.

Man könnte von einem Verfahren narrativer Enthierarchisierung sprechen, und zwar in stilistischer wie in semantischer Hinsicht. Es kommt jenes Prinzip hinzu, das ich bereits aus Anlass des ersten Kapitels genauer beschrieben habe, nämlich die Analogisierung und Spiegelung der erzählten Geschehnisse mit einem Bühnengeschehen, das gelegentlich sogar die Form eines Stationen-Dramas annimmt. Von den Rängen der Steilküsten Odessas aus beobachten große Teile der Bevölkerung und wichtige Romanpersonen das Geschehen im Hafen wie ein aufregendes Schauspiel; Jahre später empfindet der Erzähler, der durch Zufall mit der inzwischen zur kommunistischen Spionin avancierten Lika und ihrem ebenfalls geheimdienstlich aktiven Begleiter in einem teuren Petersburger Restaurant zusammentrifft, er sitze mit zwei „verkleideten Odessiten“ bzw. mit „zwei Masken“ (226) am Tisch. In der Zwischenzeit hat sich die politische Situation durch den russisch-japanischen Krieg, durch die innenpolitischen Polarisierungen erheblich verschärft, die beteiligten Personen, insbesondere die Milgrom-Tochter Lika, sind in diese Geschehnisse heftig verwickelt: aber erzählt wird im Modus von Rollen-und Kostümwechseln, von theatralen Merkwürdigkeiten und dramaturgischen Einfällen. Auf stilistischer Enthierarchisierung und semantischer Theatralisierung basiert die erinnerungs-ästhetische Besonderheit des Romans und damit das Produktionsprinzip für einen "umstrittenen Erinnerungsort".

Das bedeutet nicht, dass die tatsächlichen historischen Ereignisse zum amüsanten oder tragischen Theaterereignis degradiert würden. Es bedeutet auch nicht, dass Autor und Erzähler einem Geschichtsfatalismus das Wort redeten, der im historischen Geschehen das unbeeinflussbare Walten unbekannter Mächte oder die schiere Kontingenz erkennt. So lakonisch-amüsant, so grotesk-traurig, so heiter und zugleich düster Jabotinskys odessitischer Familienroman ist, so entschieden ist er auf ein historisches Versagen, auf ein schmerzliches Scheitern, auf eine erzählerisch nur mit äußerster Anstrengung einzuholende Zukunftshoffnung bezogen.

Ihr gelten die selbstreflexiven Passagen, die den Roman durchziehen, ihr gilt ein Kapitel, das gleichsam die Mittelachse des Romans bildet und als Kapitel 14 die Überschrift trägt: „Eingeschobenes Kapitel. Nicht für den Leser bestimmt“ (117 ff.). Es handelt vom Versuch einer Naturbeschreibung und Landschaftsschilderung, der jahreszeitlich wechselnden Farbgebung von Landschaft und Meer, von Vegetation und Klima, Krabben, Sonnenblumenkernen und Gerüchen und schließlich von der Frage, warum es eigentlich das „schwarze Meer" heiße. Der Leser erhält auf all dies eine poetisch-präzise Antwort und am Ende dieses angeblich für den Leser nicht gedachten Kapitels liest man: 
Wir spielten alle erdenklichen Spiele, mit und ohne Ball; im Übrigen habe ich auch das schon einmal irgendwo beschrieben. Wenn man ein Leben lang schreibt, geniert man sich am Ende, auch nur noch ein Wort zu sagen. Das ist furchtbar dumm. Das Leben ist dumm...aber wunderbar: Böte man mir an, es zu wiederholen - ich würde es wiederholen, haargenau so wie es war, mit allem Unglück und allen Scheußlichkeiten, wenn es nur noch einmal in Odessa beginnen könnte (122).

Das steht in der Mitte des Romans. An seinem Ende steht etwas ganz anderes; nicht sentimentales Pathos und nostalgisches Bekenntnis zum unverlierbaren Herkunftsort; sondern das realistische Eingeständnis, dass aus dem scheußlich-schönen Geschehen bühnenreif agierender Figuren in den Straßen und Plätzen Odessas, in seinen literarischen Salons und Klubs, politischen Debattierzirkeln und Redaktionsstuben, in den Häusern der vornehmen, assimilierten Juden oder der Falschspieler und Betrüger in der Moldawanka, dass - in den Worten des Erzählers - aus dem vielsprachig- hoffnungsfrohen, frühlingshaften Aufbruch einer ganzen Generation ein dumpfes Fieber wurde. Es erwuchs keineswegs nur aus mangelndem politischen Realitätssinn, es war nicht lediglich Folge generationentypischer Illusionen und überzogener Ideale, es war - so erläutert der Erzähler-Chronist - Indikator eines epochal revolutionären Prozesses. Der freilich galt gar nicht in erster Linie der Verwirklichung kommunistischer Ziele, zionistischer oder liberal-demokratischer Ideen. Der Um-und Aufbruchsprozess, der in Leben und Tod der fünf Milgrom-Kinder gespiegelt und illustriert wird, illustriert das Ende der Assimilation, den Untergang eines transnationalen Verständnisses von Judentum, den ebenso folgerichtigen wie illusionären Wunsch „zu sein wie alle Völker“ (271).

Diese zuletzt zitierte Bemerkung stammt aus dem Munde Toriks, der mit ihr seinen Entschluss zur Konversion, seine radikale Ablehnung des Zionismus begründet. Zuvor hatte sein Vater - ebenfalls im Gespräch mit dem Erzähler - eine Lesart des Hiob-Buches entwickelt, die das eigene unverdiente Schicksal in demjenigen des biblischen Helden vorgebildet sieht und damit die Frage aufwirft, wie denn auf ein solches Schicksal zu reagieren sei: mit Rebellion und Abfall von Gott oder mit "Stolz". Weder Konversion noch Zionismus liefern für Ignaz Albertowitsch die Antwort, sondern ein stolzes „Salutieren“ und Brüllen: „Gern geschehen, Euer Hochwohlgeboren“. Rebellion gegen das grundlose, empörende Handeln Gottes würde in den Augen von Vater Milgrom dem Eingeständnis gleichkommen, die erfahrenen Schicksals-Schläge seien sinnlos. Ihnen und ihrem Verursacher hingegen stolz zu salutieren heißt, sich als Teil eines Plans und gerade nicht als Objekt des Zufalls zu verstehen. Das Geschehen von der Erschaffung der Welt, über die Sintflut, die Zerstörung des Tempels, die Kreuzzüge bis zum Sturm auf die Bastille und damit auch „das Unglück im Hause des Herrn Hiob“ (265) als Teil eines Plans, als „Note in der großen Oper" zu verstehen, bedeutet: 
Also bist du keine Schnecke, du bist ein Märtyrer der Oper, ohne dich wäre der Chor unvollständig; du bist eine Person, du gehörst an die Seite ebendieses Gottes; du salutierst nicht nur ihm, sondern auch dir selbst - na ja, das alles steht hier nicht so mit diesen Worten, aber bei dem ganzen Streit geht es genau darum. Ein großartiges Buch (265).

Um die theologische Plausibilität dieser Hiob-Interpretation, die auf einen existenziellen Überlebensmodus zielt, ist es mir hier nicht zu tun. Auffällig scheint mir, dass sie nicht als einfache exegetische Erzählung gestaltet wird, sondern wiederum als ein Bühnengeschehen, in diesem Falle des großen Welttheaters, einer Verdi-Oper.

Verdis Opernwerk und Maeterlincks Dramen hatten dem Zeitgeist des Fin de siècle bekanntlich die musikalisch-dramatischen Leitbilder geliefert. Mit der Theateraufführung von „Monna Vanna“ im ersten Kapitel und der mit Bildern einer Verdi-Oper arbeitenden Hiob-Deutung am Ende seines Romans illustriert und symbolisiert Jabotinsky also ein sozial-, kultur- und mentalitätsgeschichtliches Geschehen, das im Raum einer Familie angesiedelt, am Ort großer Ereignisse situiert und als literarische Reflexion epochaler Fragen konzipiert ist. Damit aber wird Odessa bei Jabotinsky auf singuläre Weise zu einem „umstrittenen Erinnerungsort": Denn es firmiert als große Bühne, als Austragungsort von zeitgenössischen Debatten über Assimilation, Revolution, Zionismus und Konversion. Der Erinnerungsort ist nicht einfach nur „umstritten“, er ist vor allem ein Ort der Aufklärung, nicht der nostalgischen Verklärung; ein Ort, dessen Topographie und Geschichte, dessen landschaftliche und atmosphärische Besonderheiten, dessen soziale und kulturelle Milieus sich der retrospektiven Erinnerung in divergierenden, ambivalenten, heiteren und düsteren Bildern aufdrängt.

Isaac Babel, der andere große literarische Chronist, hat seine Geschichten aus Odessa bekanntlich vor allem in der Moldawanka, im jüdischen Viertel Odessas, situiert und gelegentlich behauptet „Odessa ist eine abscheuliche Stadt" (Babel 82).

Das war auf Armut und Verfolgung, auf Elend und Pogromerfahrung der Juden Odessas bezogen. Odessa „war eine komische Stadt" (281) heißt es hingegen am Ende von Die Fünf. So gegensätzlich die Aussagen, so entschieden hätte doch Babel wohl einem literarischen Befund beigepflichtet, mit dem Jabotinsky die literarische Erinnerungsarbeit seines Erzählers bilanziert: In Odessa habe man „das schönste Lied der Menschheit hören (können): hundert Sprachen“ (Jabotinskky 281).

\section{LITERATURVERZEICHNIS}

Awerbuch, Marianne. Erinnerungen aus einem streitbaren Leben. Von Berlin nach Palästina - Von Israel nach Berlin. Berlin: Hentrich \& Hentrich, 2007.

Babel, Isaak. Ein Abend bei der Kaiserin. Prosa, Reden, Tagebuch, Briefe. Neuwied: Luchterhand, 1970. 
Brumlik, Micha. Aufrichtiger Realismus: Wladimir Jabotinsky. In: Ders.: Wann, wenn nicht jetzt? Versuch über die Gegenwart des Judentums. Berlin: Neofelis, 2016, S. 70-75.

Buckard, Christian. Ein extremes Leben. Arthur Koestler 1905-1983. München: C.H.Beck, 2004.

Hohmann Andreas, Mümken Jürgen. Kischniew. Das Pogrom 1903. Lich: Edition AV, 2015.

Kaplan, Eran. Altalena. In: Enzyklopädie jüdischer Geschichte und Kultur. Band 1. Stuttgart/Weimar: Metzler, 2011, S. 50-55.

Jabotinsky, Vladimir. Die Fünf. Roman. Berlin: Aufbau, [2012] 2017.

Raabe Katharina, Sznajderman Monika. Odessa Transfer. Nachrichten vom Schwarzen Meer. Frankfurt am Main: Suhrkamp, 2009.

Schmid, Ulrich M. „Das Ende der Assimilation”. Neue Zürcher Zeitung (148) 2013.

Stanislawski, Michael. Zionism and the Fin de Siècle: Cosmopolitanism and Nationalism from Nordau to Jabotinsky. Berkeley: University of California Press, 2001. 
